\title{
Efectos de la legalización del aborto en Nepal 2001 - 2010 (1)
}

\author{
Henderson JT, Puri M, Blum M, Harper CC, Rana A, et al. Effects of abortion legalization \\ in Nepal, 2001-2010. PLoS ONE 2013;8(5):e64775.
}

Análisis crítico: Claudio Vera $P-G^{1,2}, M S c$. Jorge Carvajal $C^{1}, P h D$.

${ }^{1}$ Unidad de Medicina Materno-Fetal, División de Obstetricia y Ginecología; ${ }^{2}$ Unidad de Medicina Basada en Evidencia. Escuela de Medicina. Pontificia Universidad Católica de Chile

\section{RESUMEN (1)}

Antecedentes: El aborto fue legalizado en Nepal el año 2002, luego de esfuerzos de promoción de la salud resaltados por una alta mortalidad materna proveniente de aborto inseguro. Buscamos evaluar si la legalización conlleva reducciones de las consecuencias más graves del aborto inseguro. Métodos: Conducimos una revisión retrospectiva de fichas clínicas de todos los casos ginecológicos que se presentaron en cuatro hospitales de referencia en Nepal. Para los años 2001-2010, todos los casos de complicaciones de aborto espontáneo e inducido fueron identificados, resumidos y codificados para clasificar los casos de infección grave, lesiones o complicaciones sistémicas. Usamos regresión segmentada de Poisson y regresión logística ordinaria para evaluar la tendencia y riesgo de las complicaciones graves en tres periodos: antes de la implementación (2001-2003), implementación temprana (2004-2006) e implementación tardía (2007-2010). Resultados: 23.493 casos de complicaciones de aborto fueron identificados. Una tendencia significativa a la reducción de la proporción de infección grave, lesiones y complicaciones sistémicas se observó en el periodo de implementación tardía, junto con una declinación en el riesgo de complicaciones graves (OR: 0,7; IC95\% 0,64 a 0,85). Reducciones en la sepsis ocurrieron más temprano, durante el periodo de implementación precoz (OR: 0,6; IC95\% 0,47 a 0,75). Conclusión: En el periodo de estudio, el uso de atención de salud y la población en edad reproductiva aumentó. La fertilidad total también declinó a la mitad, no obstante una prevalencia de uso contraceptivo. Un mayor número de mujeres habrían abortado y buscado atención hospitalaria por complicaciones luego de la legalización, así observamos una reducción significativa en la tasa de morbilidad grave por aborto. La liberalización de la política de aborto en Nepal ha beneficiado a la salud de la mujer, y contribuiría a la reducción de la mortalidad materna en el país. La caída más pronunciada se observó luego de la expansión del programa de aborto seguro para incluir proveedores de nivel medio, entrenamiento en segundo trimestre y medicación abortiva, resaltando la importancia de concentrar los esfuerzos para mejorar el acceso. Otros países que contemplan cambios en su política de aborto pueden basarse en la evidencia y estrategias de implementación observadas en Nepal.

\section{ANÁLISIS DE LA INVESTIGACIÓN}

\section{A. Relevancia clínica de la investigación}

En Chile el aborto provocado es ilegal (2), políticas sobre diferentes tipos de aborto legal han sido debatidas (3), la evidencia sobre potenciales beneficios en la salud materna es considerada en su favor. Un estudio reciente sobre la experiencia de la legalización del aborto en Nepal evalúa sus efectos (1).

Escenario clínico: Nepal es una nación asiática multiétnica ubicada entre China e India, comparte con sus vecinos una alta densidad de habitantes. Con una población estimada para el año 2001 de 23 millones de habitantes (4). Presentó una guerra civil desde fines de los noventa hasta el año 2006 con importantes consecuencias políticas y económicas, con una lenta recuperación pese a los esfuerzos de asistencia internacional. Previo al año 
2002, el aborto en Nepal tenía un estatus legal similar al homicidio, con pena de presidio. La tasa de mortalidad materna de Nepal para el año 2000 se estimó en 360 por 100.000 NV con una progresiva disminución, estimándose cercana a 200 por $100.000 \mathrm{NV}$, donde menos del $20 \%$ de los partos tienen atención profesional. Diversas estrategias se han implementado en Nepal con el objetivo de reducir la mortalidad materna (5). El aborto fue legalizado el año 2003 (1).

\section{B. El estudio (1)}

Diseño: Estudio retrospectivo de revisión de fichas. Pacientes: Todos los ingresos desde enero de 2001 hasta diciembre de 2010, en 4 hospitales mayores de referencia con población asignada de bajos ingresos. En conjunto los hospitales asisten aproximadamente al $38 \%$ de la población de $\mathrm{Ne}$ pal. Evaluación de los eventos: Los autores revisaron todos los ingresos y los casos de mortalidad materna y neonatal que se presentan en todas las unidades de los hospitales incluidos, en base a protocolos preestablecidos refinados con pilotos previos. Durante la recolección de datos muestras aleatorias de la información original fue evaluada con la ficha clínica para asegurar la exactitud de la información. Inconsistencias fueron corregidas previo al análisis. Resultados medidos: El evento de interés del estudio fueron las complicaciones graves relativas a todas las complicaciones por aborto. Los autores incluyeron tanto las complicaciones relacionadas a aborto espontáneo como inducido debido a la dificultad en diferenciarlos apropiadamente. Además se evaluaron las complicaciones por aborto relativas a los nacidos vivos en el periodo de tiempo. La severidad de las complicaciones fueron categorizadas con esquema simplificando de esquemas disponibles para distinguir los casos de mayor gravedad de infección, lesión o complicaciones sistémicas (muerte, pérdida de conciencia, falla orgánica, cuerpo extraño o lesión por aborto, infección pélvica o del tracto genital, endometritis, peritonitis local o generalizada, shock hipovolémico, shock séptico o septicemia, pulso de 120x', temperatura mayor de $38,9^{\circ} \mathrm{C}$ ). Las complicaciones fueron evaluadas en 3 periodos: antes de la implementación (2001-2003), implementación precoz (2004-2006), e implementación tardía (2007-2010). Los tipos de complicaciones fueron analizados en los 3 periodos. Resultados: De 24.676 casos potenciales finalmente se incluyeron 23.493 casos de complicaciones de aborto para su análisis. La edad promedio de las mujeres que presentaron un aborto fue 25,4 años, nulíparas $32,2 \%$. El aborto inducido fue reportado en globo en $9,6 \%$ de los casos. El número total de complicaciones relacionadas con aborto aumentó de 6.486 en el primer periodo a 10.144 en el último periodo. La proporción glo- bal de complicaciones graves asociadas a aborto (relativa a las complicaciones asociadas a aborto) fue menor en el periodo de implementación tardía. El análisis multivariable, sobre el riesgo de complicaciones graves para cada periodo, muestra una reducción significativa del riesgo en el último periodo comparado con el primero (OR: 0,74; IC95\% 0,64 a 0,85), una mayor reducción se observó en el subgrupo de aborto inducido (OR: 0,49; IC95\% 0,37 a 0,64). El riesgo de sepsis fue menor en el periodo de implementación tardía. La edad materna y la edad gestacional al aborto se asociaron con mayor riesgo de complicaciones graves, así como el aborto inducido. Se observó una tendencia significativa a la disminución de complicaciones graves asociadas a aborto cuando se analizó como proporción de los nacidos vivos en 2 de los hospitales con datos disponibles.

\section{Análisis crítico}

Validez interna: El propósito del estudio fue evaluar las complicaciones graves de aborto en un periodo de 10 años con la hipótesis de que declinan, en los mayores hospitales de referencia en Nepal, luego de la legalización del aborto. El tipo de estudio seleccionado para evaluar la hipótesis corresponde a series de casos retrospectivo, con comparación de la prevalencia de eventos graves entre 3 periodos de implementación. Selección de la población, representatividad: Dado la naturaleza retrospectiva de selección de casos del estudio, no es posible conocer si la muestra es representativa de la situación global de Nepal con respecto a las complicaciones por aborto. El estudio posiblemente representa a pacientes con eventos que se presentaron en los hospitales de referencia más grandes de Nepal. No representa a la población que no accedió a los hospitales, no hay búsqueda de eventos en otras fuentes como certificados de defunción. Evaluación de los eventos de interés: Los autores implementan un protocolo estandarizado con mejoramiento iterativo para la selección de los eventos. No es posible estimar la proporción de eventos no pesquisados por el protocolo, a pesar de que se describen auditorías a las fichas de extracción de datos. No se describen las causas de exclusión de fichas potenciales. Los resultados están expresados en tasas de complicaciones graves relativas a las complicaciones por aborto, no es posible conocer cuantas pacientes presentaron 2 o más eventos ni la tasa de complicaciones graves por paciente. El análisis no incluye comparaciones entre cada par de periodos por lo cual, el hallazgo de al menos una proporción diferente a lo esperado con el test exacto de Fisher no necesariamente informa sobre cual es la menor o si esto tiene sentido en base a la hipótesis planteada. Así por ejemplo, por un lado llama la atención que las lesiones asociadas 
Tabla I

EVOLUCIÓN DE LAS COMPLICACIONES GRAVES ASOCIADAS A ABORTO

\begin{tabular}{lcccc}
\hline Complicaciones graves & $\begin{array}{c}\text { Periodo 1 } \\
\text { Eventos por 1.000 } \\
\text { complicaciones aso- } \\
\text { ciadas a aborto }\end{array}$ & $\begin{array}{c}\text { Periodo 2 } \\
\text { Eventos por 1.000 } \\
\text { complicaciones } \\
\text { asociadas a aborto }\end{array}$ & $\begin{array}{c}\text { Periodo } 3 \\
\text { Eventos por 1.000 } \\
\text { complicaciones } \\
\text { asociadas a aborto }\end{array}$ & ${ }^{*}$ Valor $\mathrm{p}$ \\
\hline Muerte & 0,6 & 0,6 & 0,4 & $\mathrm{NS}$ \\
Infecciones & 50,6 & 42,3 & 42,2 & $<0,05$ \\
Lesiones & 2,2 & 6 & 4,4 & $<0,001$ \\
Sistémicas & 28,7 & 35 & 23,6 & $<0,001$ \\
Cualquier complicación grave & 73,4 & 71,1 & 62,5 & $<0,05$ \\
\hline
\end{tabular}

*Test exacto de Fisher

a aborto (incluyendo lesiones uterinas, vaginales perineales, intestinales, cervicales y cuerpos extraños) aumentaron significativamente de 2,2 por 1.000 complicaciones en el primer periodo a 5 por 1.000 en los periodos posteriores $(p<0,001)$. Por otro lado, en las complicaciones agrupadas como sistémicas (frecuencia cardiaca elevada, shock, falla renal, falla multiorgánica, coagulación intravascular diseminada, síndrome de distrés respiratorio, falla cardiaca, otras sistémicas) si bien, al menos una tasa es diferente a lo esperado en base al resultado global, llama la atención que las complicaciones aumentaron en el segundo periodo y disminuyen en el tercer periodo por lo cual, no es posible definir si es el aumento en el segundo periodo el que define la significancia estadística para este evento, o si existe diferencia entre el primero y el último periodo. No se observaron diferencias estadísticamente significativas en las muertes maternas. Importantes fuentes de sesgo y limitaciones en un estudio descriptivo deben ser consideradas al interpretar los resultados. Los autores realizan una exhaustiva descripción de las potenciales limitaciones y amenazas a la validez entre las cuales destacan cambios demográficos, sociales y políticos. Así también, mejorías de acceso a sistemas de salud, entrenamiento del equipo de salud junto con disponibilidad de medicación en relación a aborto pueden haber afectado el perfil de complicaciones hacia uno de menor gravedad sobretodo en el último periodo. Los autores reconocen la dificultad de obtener datos de buena calidad en un escenario de bajos recursos, y de cómo cambios en las prácticas de documentación en las fichas podrían haber afectado la calificación de la severidad de los casos.

\section{COMENTARIO}

Este estudio con importantes limitaciones describe una reducción de la tasa global de compli- caciones graves relativas a las complicaciones asociadas a aborto de 3 hospitales importantes de Nepal en el periodo tardío de la implementación del aborto legal. Tanto los contextos locales sociales y políticos como los cambios en implementación de medicación asociada al manejo del aborto pueden haber afectado el perfil de complicaciones asociadas a aborto (6). Si bien, los esfuerzos a múltiples niveles han provocado una dramática caída de la mortalidad materna en Nepal en las últimas 2 décadas, aún se estima que estará sobre 100 por 100.000 NV para el año 2015 (7). Dado el contexto específico de esta nación durante el periodo en estudio y las diversas limitaciones y consideraciones que pueden influir en la reducción observada, consideramos que los resultados observados posterior a la legalización del aborto en los hospitales evaluados de Nepal son difícilmente extrapolables a otros escenarios.

\section{REFERENCIAS}

1 Henderson JT, Puri M, Blum M, Harper CC, Rana A, Gurung $\mathrm{G}$, et al. Effects of abortion legalization in $\mathrm{Ne}$ pal, 2001-2010. PLoSOne 2013;8(5):e64775.

2 Ministerio de Justicia. Código Penal. Crímenes y Delitos contra el orden Familiar y la Moralidad Pública: Artículos 342 a 345. 1874. Disponible en: http://www. leychile.cl/N? $\mathrm{i}=1984 \& \mathrm{f}=2013-08-20 \& p=$. Acceso el 25 de agosto de 2013.

3 Biblioteca del Congreso Nacional. Aborto terapéutico: hitos legislativos. 2013. Mirada en profundidad. Disponible en: http://www.bcn.cl/carpeta_temas_profundidad/aborto-terapeutico-hitos-legislativos. Acceso el 26 de agosto de 2013.

4 Central Bureau of Statistics. Nepal in Figures 2012. 2012. Disponible en: http://cbs.gov.np/wp-content/ uploads/2012/Nepal\%20in\%20figure/Nepal\%20 In\%20Figures\%202012_English.pdf. Acceso el 18 de agosto de 2013. 
5 Nepal Family Health Program II. Community-Based Postpartum Hemorrhage Prevention. Technical Brief \#11. (Revised June 2010). Disponible en: http://nfhp. jsi.com/Res/Docs/TechBrief11-PPH.pdf. Acceso el 18 de agosto de 2013.
6 Rocca C, Puri M, Dulal B, Bajracharya L, Harper C, Blum $\mathrm{M}$, et al. Unsafe abortion after legalisation in $\mathrm{Ne}-$ pal: a cross-sectional study of women presenting to hospitals. BJOG 2013;120(9):1075-84.

7 Bhandari TR, Dangal G. Maternal Mortality: Paradigm shift in Nepal. NJOG 2012;7(2):3-8. 